\title{
Efficient Synthesis of PbTe Nanoparticle Networks
}

\author{
Qiangfeng Xiao ${ }^{1}$, Ding Weng ${ }^{1}$, Zhenglong Yang ${ }^{1}$, Javier Garay ${ }^{2}$, Minjuan Zhang ${ }^{3}(\varangle)$, and Yunfeng Lu ${ }^{1}(\varangle)$ \\ ${ }^{1}$ Chemical and Biomolecular Engineering Department, University of California, Los Angeles, CA 90095, USA \\ ${ }^{2}$ Mechanical Engineering Department, University of California, Riverside, CA 92521, USA \\ ${ }^{3}$ Materials Research Department, Toyota Motor Engineering and Manufacturing North America, Inc., 1555 Woodridge Ave. Ann Arbor, \\ MI 48105, USA
}

Received: 23 April 2010 / Revised: 17 July 2010 / Accepted: 6 August 2010

(C) The Author(s) 2010. This article is published with open access at Springerlink.com

\begin{abstract}
The synthesis of semiconductor nanocrystalline networks using weak capping ligands in aqueous media has been demonstrated. Carbohydrates, including $\beta$-cyclodextrin, $D$-(+)-glucose, $D$-glucosamine, lactobionic acid, sucrose, and starch were chosen as weak ligands to facilitate the formation of PbTe nanoparticle networks. The nanoparticle size, ranging from $5 \mathrm{~nm}$ to $30 \mathrm{~nm}$, can be tuned by manipulating the temperature and concentration. Through a similar strategy, more complicated nanostructures including carbohydrate spheres@PbTe core-shell structures and Te@carbohydrate@PbTe multilayered submicron cables have been fabricated. This is a general approach which can be easily extended to the fabrication of other semiconductor networks, including PbSe and $\mathrm{Bi}_{2} \mathrm{Te}_{3}$ using carbohydrates and ethylenediaminetetraacetic acid (EDTA), respectively, as ligands.
\end{abstract}

\section{KEYWORDS}

Lead telluride, nanoparticle, network, core shell structures, thermoelectric, carbohydrate

\section{Introduction}

Compared to the bulk materials, nanocrystals possess unique size-dependent chemical and physical properties due to their quantum confinement effect [1]. Great success in the synthesis of colloidal nanocrystals with controlled morphology and composition has been achieved in the last two decades [2-5]. More advanced one-, two-, and three-dimensional superstructures have been constructed for practical device applications using nanocrystals as building blocks. For example, oriented attachment of preformed nanocrystals allows the formation of one-dimensional nanostructures of various materials, such as $\mathrm{Ag}$ [6], $\mathrm{ZnO}$ [7], $\mathrm{ZnS}$ [8], PbSe [9], PbTe [10], and CdTe [11]. Two-dimensional nematic and smectic liquid-crystalline phases [12] have been obtained by Langmuir-Blodgett assembly of $\mathrm{BaCr}_{2} \mathrm{O}_{4}$ nanorods, whilst a water-droplet assisted self-assembly of nanocrystals generated various combinatorial hierarchically ordered two-dimensional architectures [13]. Three-dimensional ordered superlattices can be obtained using mixed solvents [14], programmable deoxyribonucleic acid (DNA) ligands $[15,16]$, or external magnetic fields [17]. Furthermore, the combination of binary monodisperse nanocrystals of different sizes and interactions can yield a wide range of three-dimensional superlattices with tunable structures and properties [18, 19].

Nanocrystalline networks facilitate charge transport and possess large surface area. Such materials may

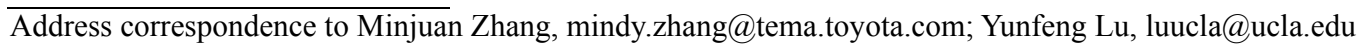


find applications in catalysis, sensors, solar cells, and other fields. Generally, nanocrystalline networks can be prepared by either template or template-free methods. Of these methods, construction of nanocrystalline networks from preformed nanocrystals is attracting increasing interest. For instance, Au networks were prepared from $\mathrm{Au}$ particles by hydrothermally detaching surfactants [20], and CdS, ZnS, PbS, CdSe, and CdTe semiconductor networks (aerogels) were fabricated by partial oxidative ligand removal from nanoparticles synthesized in either aqueous or organic solution [21, 22]. PbSe nanocrystal networks have also been formed by the displacement of oleic acid ligands with pyridine [23]. In order to circumvent the necessity of removing the template or the synthesis of preformed nanocrystals, we have devised a one-step strategy for the synthesis of nanocrystalline networks using weak ligands. Various carbohydrates, such as $\beta$ cyclodextrin, $D$-(+)-glucose, $D$-glucosamine, lactobionic acid, sucrose, and starch were chosen as weak ligands. Compared to anchoring points such as $-\mathrm{COOH}$, $-\mathrm{PO}_{3} \mathrm{H}_{2},-\mathrm{SH},-\mathrm{NH}_{2}$, and $\equiv \mathrm{P}=\mathrm{O}$ in commonly used ligands, the binding capacity of $-\mathrm{OH}$ on the carbohydrates is relatively weak. The weak ligands lack strong the steric repulsion and binding capability required to produce isolated nanoparticles. Therefore the growing nanoparticles tend to form continuous networks in order to reduce their surface energy. Among various semiconductors, lead telluride ( $\mathrm{PbTe})$ is of particular interest owing to its unique combination of properties including large excitonic Bohr radius, high carrier mobility, efficient multiexciton generation, large dielectric constant, high melting point, low vapor pressure, and relatively high thermoelectric figure of merit [24-27]. Although PbTe nanoparticles, nanorods, and nanowires have previously been produced, this work represents the first synthesis of nanocrystalline networks of this material.

\section{Experimental}

\subsection{Reagents}

Lead(II) nitrate (99.999\%), sodium hydroxide (beads, 20-40 mesh), potassium hydroxide ( $\geqslant 85 \%)$, tellurium (powder), sodium borohydride ( 98\%), hydrazine (anhydrous), polyvinylpyrrolidone (average $M_{\mathrm{w}}$ $\sim 55,000), \beta$-cyclodextrin (powder), $D$-(+)-glucose $(\geqslant 99.5 \%)$, sucrose $(\geqslant 99.5 \%)$, starch (soluble, ACS reagent), $\alpha$-lactose monohydrate (reagent grade), $D$-(+)-glucosamine hydrochloride $(\geqslant 99 \%)$, lactobionic acid (97\%), poly(ethyleneimine) solution (PEI, average $M_{\mathrm{n}} \sim 1,200$ ), ethylenediaminetetraacetic acid $(99 \%)$, and 3-mercaptopropionic acid ( $\geqslant 99 \%)$ were purchased from Aldrich.

\subsection{Synthesis of PbTe networks}

NaHTe was prepared [28] by reacting sodium borohydride with tellurium in water at $0{ }^{\circ} \mathrm{C}$ for $8 \mathrm{~h}$ using a molar ratio of sodium borohydride:tellurium:water $=$ 2:1:56. The lead precursors were prepared by consecutively dissolving $2 \mathrm{~g}$ of $\mathrm{KOH}, 1 \mathrm{~g}$ of carbohydrate, and $1.655 \mathrm{~g}$ of $\mathrm{Pb}\left(\mathrm{NO}_{3}\right)_{2}$ in $10 \mathrm{~mL}$ of distilled (DI) water under sonication. After purging the carbohydrate- $\mathrm{Pb}$ solution with nitrogen, an equimolar amount of freshly prepared NaHTe solution was added dropwise at room temperature under stirring, resulting in the immediate formation of black PbTe nanoparticles. The nanoparticles were separated by centrifugation, washed with ethanol and water, and finally dried under vacuum. This synthesis protocol is highly effective. For example, $10 \mathrm{~g}$ or more of PbTe nanoparticles can be prepared in less than 20 min starting with $12 \mathrm{~g}$ of $\mathrm{Pb}\left(\mathrm{NO}_{3}\right)_{2}$ in a $250 \mathrm{~mL}$ flask with a high yield of $80 \%$.

\subsection{Synthesis of carbohydrate sphere @PbTe core- shell structures}

Carbohydrate spheres were prepared [29-32] by dissolving $4 \mathrm{~g}$ of $\mathrm{D}$-(+)-glucose in $100 \mathrm{~mL}$ of distilled water and treating the resulting solution hydrothermally at $190{ }^{\circ} \mathrm{C}$ for $4 \mathrm{~h}$. The resulting dark brown precipitate was isolated by centrifugation and purified by three cycles of centrifugation/washing/redispersion in water and then in alcohol. Then $0.1 \mathrm{~g}$ of the as synthesized carbohydrate spheres, $0.02 \mathrm{~g}$ of $\mathrm{NaOH}$, and $0.8275 \mathrm{~g}$ of $\mathrm{Pb}\left(\mathrm{NO}_{3}\right)_{2}$ were added to $30 \mathrm{~mL}$ of distilled water and the mixture ultrasonicated for $20 \mathrm{~min}$. The resulting suspension was separated by centrifugation/washing/redispersion in water and 
then in alcohol, and redispersed in $30 \mathrm{~mL}$ of distilled water. Under a flow of $\mathrm{N}_{2}, 1 \mathrm{~mL}$ of $0.5 \mathrm{~mol} / \mathrm{L} \mathrm{NaHTe}$ solution was added dropwise at room temperature over $10 \mathrm{~min}$. The carbohydrate composite spheres were obtained by another three cycles of centrifugation/ washing/redispersion in water and alcohol.

\subsection{Synthesis of Te@ carbohydrate@PbTe multilayer cable structures}

Te nanowires were obtained [33] by dissolving $0.6 \mathrm{~g}$ of polyvinylpyrrolidone (PVP), $0.1107 \mathrm{~g}$ of $\mathrm{Na}_{2} \mathrm{TeO}_{3}$, $1 \mathrm{~mL}$ of hydrazine hydrate $(85 \% \mathrm{w} / \mathrm{w})$, and $2 \mathrm{~mL}$ of an aqueous ammonia solution $(25 \%-28 \% \mathrm{w} / \mathrm{w})$ in $22 \mathrm{~mL}$ of distilled water and hydrothermally treating the mixture at $180{ }^{\circ} \mathrm{C}$ for $4 \mathrm{~h}$. The Te@carbohydrate cables were prepared by dissolving $2 \mathrm{~g}$ of $\mathrm{D}$-(+)-glucose in $100 \mathrm{~mL}$ of distilled water with $0.1 \mathrm{mmol}$ of Te nanowires as the template and hydrothermally treating the mixture at $180^{\circ} \mathrm{C}$ for $8 \mathrm{~h}$. The precipitate was isolated by centrifugation and purified by three cycles of centrifugation/washing/redispersion in water and then in alcohol. Then $0.1 \mathrm{~g}$ of as synthesized Te@carbohydrate cable, $0.02 \mathrm{~g}$ of $\mathrm{NaOH}$, and $0.8275 \mathrm{~g}$ of $\mathrm{Pb}\left(\mathrm{NO}_{3}\right)_{2}$ were added to $30 \mathrm{~mL}$ of distilled water and the mixture stirred magnetically for $20 \mathrm{~min}$. The resulting suspension was separated by centrifugation/ washing/redispersion in water and then in alcohol, and redispersed in $30 \mathrm{~mL}$ of distilled water. Under a flow of $\mathrm{N}_{2}, 1 \mathrm{~mL}$ of $0.5 \mathrm{~mol} / \mathrm{L} \mathrm{NaHTe}$ solution was added dropwise at room temperature over $10 \mathrm{~min}$. Finally the carbohydrate composite spheres were obtained by three cycles of centrifugation/washing/ redispersion in water and then in alcohol.

\subsection{Characterization}

Powder X-ray diffraction (XRD) patterns were obtained using a PANalytical X'Pert PRO X-ray diffractometer. Transmission electron microscope (TEM) images and energy dispersive spectra (EDS) were obtained using a JEOL 2011 FasTEM microscope. For X-ray photoelectron spectroscopy (XPS) measurements the samples were coated on carbon tape and the data were collected with a PHI 3057 spectrometer. Fourier transform infrared (FTIR) spectra were obtained using a JASCO FTIR spectrometer.

\section{Results and discussion}

Figure 1(a) shows a representative TEM micrograph of the sample synthesized using $1 \mathrm{~mol} / \mathrm{L} \mathrm{NaHTe}$ and $0.5 \mathrm{~mol} / \mathrm{L} \mathrm{Pb}-\beta$-cyclodextrin complex at room temperature which clearly shows that the product consists of an interconnected network of nanoparticles with diameters around $20 \mathrm{~nm}$. A high-resolution TEM (HRTEM) image of a constituent particle (Fig. 1(b)) shows the material has a crystalline structure with an adjacent lattice-fringe distance of ca. $3.2 \AA$, which is consistent with the (200) interplanar distance (3.23 $\AA$ ) of the face centered cubic (FCC) PbTe. EDS (Fig. S-1 in the Electronic Supplementary Material (ESM)) indicates a $\mathrm{Pb} / \mathrm{Te}$ atomic ratio of $1: 1$, further confirming the formation of $\mathrm{PbTe}$. Reducing the precursor concentration leads to the formation of networks composed of smaller particles. For example, Fig. 1(c) shows a TEM image of the nanoparticle network prepared using $0.1 \mathrm{~mol} / \mathrm{L} \mathrm{NaHTe}$ and $0.05 \mathrm{~mol} / \mathrm{L} \mathrm{Pb}-\beta$-cyclodextrin complex solutions. Networks of nanoparticles with an average diameter of $5 \mathrm{~nm}$ are clearly observed. It is worth mentioning that although the particle diameters are so small, these networks are crystalline (see the HRTEM image in Fig. 1(e)). Moreover, the diameter of the component particles can be further tuned by varying the reaction temperature. Figure 1(d) shows a TEM image of the nanoparticle networks prepared by the addition of $1 \mathrm{~mol} / \mathrm{L} \mathrm{NaHTe}$ to a refluxing solution of $0.5 \mathrm{~mol} / \mathrm{L} \mathrm{Pb}-\beta$-cyclodextrin complex. Networks of particles with an average diameter of $30 \mathrm{~nm}$ were formed. In general, the particle size increases with increasing concentration of the precursors as more material is available for growth, and also with increasing temperature as the growth rate increases. The ability to tune the size of the component particles potentially provides the possibility of tuning the quantum effect, which is critical to achieve an enhanced thermoelectric figure of merit. The FTIR spectra (Fig. S-2 in the ESM) indicate that $\beta$-cyclodextrin acts as a capping ligand and endows the networks with high dispersibility in water. Due to the weak binding interactions, most of the $\beta$-cyclodextrin can be removed by copious washing with water as shown by the Infrared (IR) spectra in Fig. S-2 (in the ESM). It is interesting to note that similar network structures can be formed by replacing

\section{Springer}




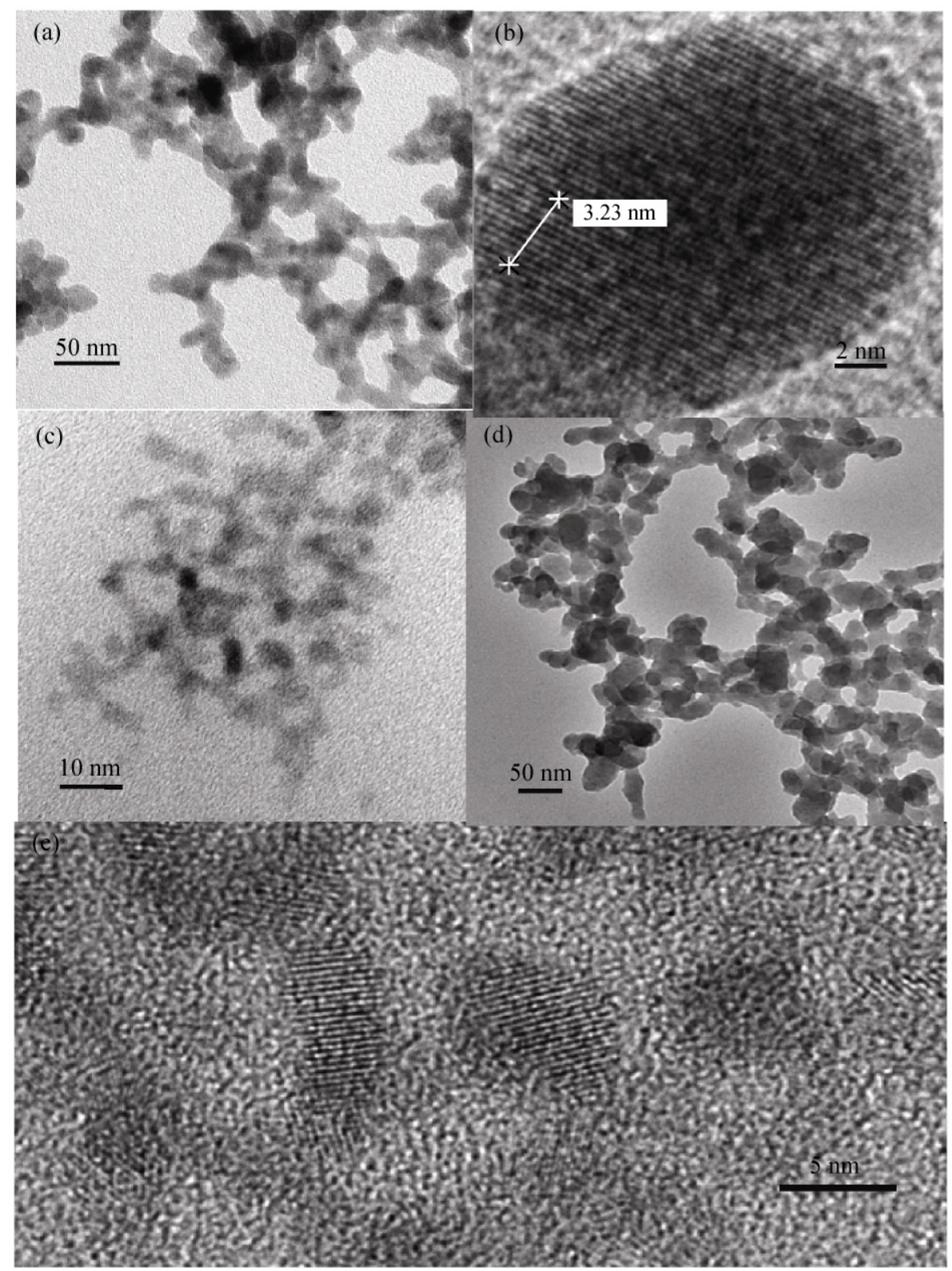

Figure 1 TEM images of the PbTe nanoparticle network prepared by reacting $1 \mathrm{~mol} / \mathrm{L}$ NaHTe with $0.5 \mathrm{~mol} / \mathrm{L} \mathrm{Pb}-\beta$-cyclodextrin complex precursor solution at room temperature $(\mathrm{a}, \mathrm{b})$ and under reflux $(\mathrm{d})$, and of that prepared by reacting $0.1 \mathrm{~mol} / \mathrm{L} \mathrm{NaHTe}$ solution with $0.05 \mathrm{~mol} / \mathrm{L} \mathrm{Pb}-\beta$-cyclodextrin complex precursor solution at room temperature $(\mathrm{c}, \mathrm{e})$

$\beta$-cyclodextrin with other low-cost carbohydrates, such as $D$-(+)-glucose (Fig. S-3 in the ESM), sucrose (Fig. S-4 in the ESM), D-glucosamine (Fig. S-5 in the ESM), lactobionic acid, and starch.

Figures 2(a) and 2(b), respectively, show the XRD patterns of the nanoparticle networks prepared from $1 \mathrm{~mol} / \mathrm{L} \mathrm{NaHTe}$ and $0.5 \mathrm{~mol} / \mathrm{L} \mathrm{Pb}-\beta$-cyclodextrin complex solutions and $0.1 \mathrm{~mol} / \mathrm{L} \mathrm{NaHTe}$ and $0.05 \mathrm{~mol} / \mathrm{L}$ $\mathrm{Pb}-\beta$-cyclodextrin complex solutions. Both patterns can be indexed to an FCC structure ( $F m 3 m$ with a unit cell parameter of $6.459 \AA$ ), which is consistent with the standard pattern of bulk PbTe. The diffraction peaks are broader than those of the bulk sample, in accordance with their smaller crystalline domains. Consistent with the TEM observations, decreasing the precursor concentration results in broader diffraction peaks, indicating smaller crystalline domain sizes. The crystallite size estimated from the Scherrer equation, 


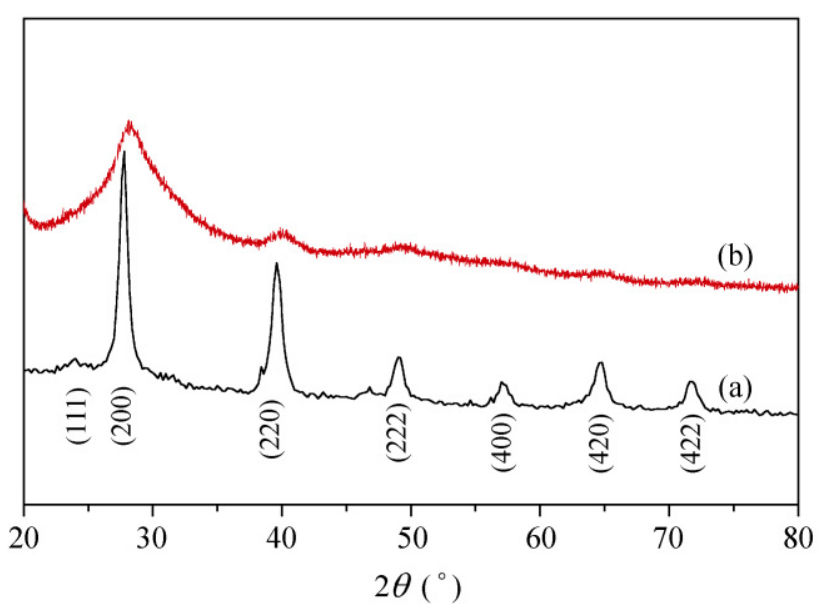

Figure 2 Powder X-ray diffraction (XRD) patterns of $\mathrm{PbTe}$ nanoparticle networks prepared from (a) $1 \mathrm{~mol} / \mathrm{L} \mathrm{NaHTe}$ and $0.5 \mathrm{~mol} / \mathrm{L} \mathrm{Pb}-\beta$-cyclodextrin complex solution and (b) $0.1 \mathrm{~mol} / \mathrm{L}$ $\mathrm{NaHTe}$ and $0.05 \mathrm{~mol} / \mathrm{L} \mathrm{Pb}-\beta$-cyclodextrin complex solution at room temperature

based on Gaussian fitting of the (200) peak, is around $20 \mathrm{~nm}$ and $8 \mathrm{~nm}$ for the particles prepared from the concentrated (Fig. 2(a)) and dilute (Fig. 2(b)) solutions, respectively. It is interesting to note that the peaks shift toward higher angles with the decrease of crystallite size, which can be ascribed to surface stress; a similar effect has been observed for gold and ionic crystals [34]. XPS (Fig. S-6 in the ESM) further confirms the formation of PbTe. XPS also shows that, on exposure to air, the surface of the PbTe networks can be easily oxidized to $\mathrm{PbTeO}_{3}$ (Fig. S-7 in the ESM).

This simple synthesis process can be extended to the construction of nanostructured materials with more complicated structures. For example, as shown in Fig. 3(a), we first prepared carbohydrate spheres with an average diameter around $100 \mathrm{~nm}$ by hydrothermally reacting an aqueous solution of $D-(+)$-glucose at $190{ }^{\circ} \mathrm{C}$ for $4 \mathrm{~h}$. We then complexed $\mathrm{Pb}^{2+}$ with these carbohydrate spheres, using a similar technique to that described above. Subsequent reaction of the complexed spheres with NaHTe afforded composite spheres containing the nanoparticle shells (Fig. 3(b)).

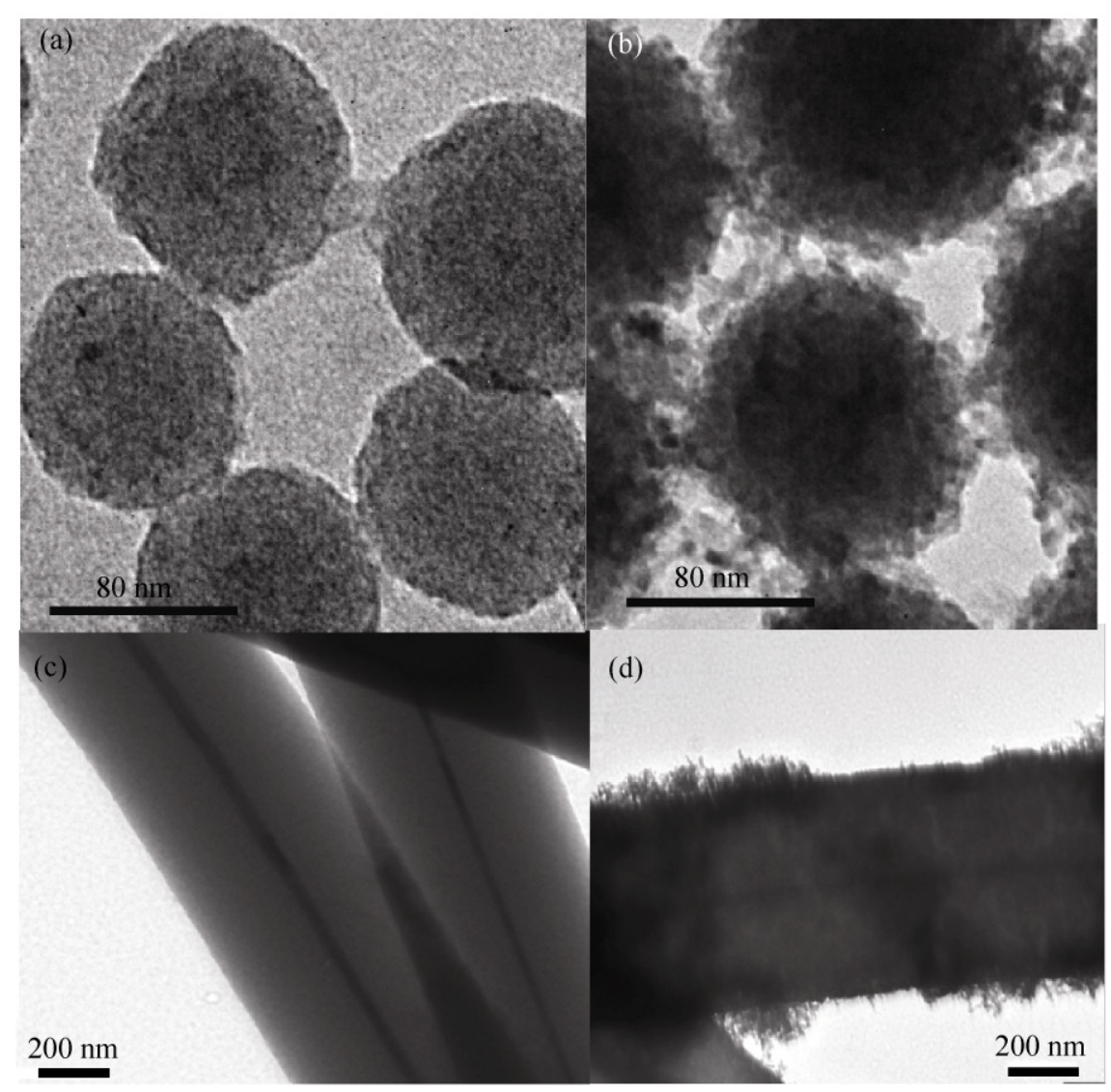

Figure 3 TEM images of (a) carbohydrate spheres, (b) carbohydrate spheres coated with PbTe nanoparticle shells, (c) Te@carbohydrate cables, and (d) Te@carbohydrate cable@PbTe multilayered submicron cables 
The formation of the PbTe shell was validated by EDS (Fig. S-8 in the ESM) and XRD (Fig. S-9 in the ESM). Using a similar strategy, Te@carbohydrate submicrocables and Te@carbohydrate tubes@PbTe multilayer submicrocables were obtained, as shown by the TEM images in Figs. 3(c) and 3(d) and the XRD patterns in Figs. S-10 and S-11 (in the ESM).

Besides carbohydrates, ethylenediaminetetraacetic acid (EDTA), thioglycolic acid (TGA), and 3-mercaptopropionic acid (MPA) were used as ligands to produce $\mathrm{PbTe}$ using the same concentration as for $\beta$-cyclodextrin. XRD and TEM (Figs. S-12 and S-13 in the ESM) showed that PbTe nanoparticle networks were also obtained. As indicated by the formation of $\mathrm{HSCH}_{2} \mathrm{CH}\left(\mathrm{NH}_{2}\right) \mathrm{COO}-\mathrm{Pb}-\mathrm{OH}$ in the reaction of $\mathrm{Pb}^{2+}$ and cysteine under weakly basic conditions, $\mathrm{Pb}^{2+}$ ions have a stronger interaction with - $\mathrm{COO}$ than with $-\mathrm{SH}$ groups [35], in contrast to the case for $\mathrm{Cd}^{2+}$. We believe that solvation of $\mathrm{Pb}^{2+}$ in aqueous media attenuates the binding capability of - $\mathrm{COO}$ with $\mathrm{Pb}^{2+}$, and consequently leads to the formation of networks. In addition to the steric repulsions caused by the capping ligands, electrostatic repulsions resulting from charged species at the surface have frequently been employed for nanoparticle stabilization. When positively charged polymeric PEI was employed as a ligand, we obtained isolated PbTe nanoparticles, as shown by XRD and TEM (Fig. S-14 in the ESM).

Based on the above results, a reaction mechanism can be proposed as illustrated in Fig. 4. The formation of $\mathrm{PbTe}$ nanoparticles, their subsequent aggregation, and growth into a continuous network structure are all involved. It is known that $\mathrm{Pb}^{2+}$ is precipitated from basic aqueous solution as $\mathrm{Pb}(\mathrm{OH})_{2}$. Adding carbohydrates-which can be deprotonated in a strongly basic environment-results in the formation of stable polyolato complexes [36]. Such complexes are present as a clear solution (Step I). Rapid reaction

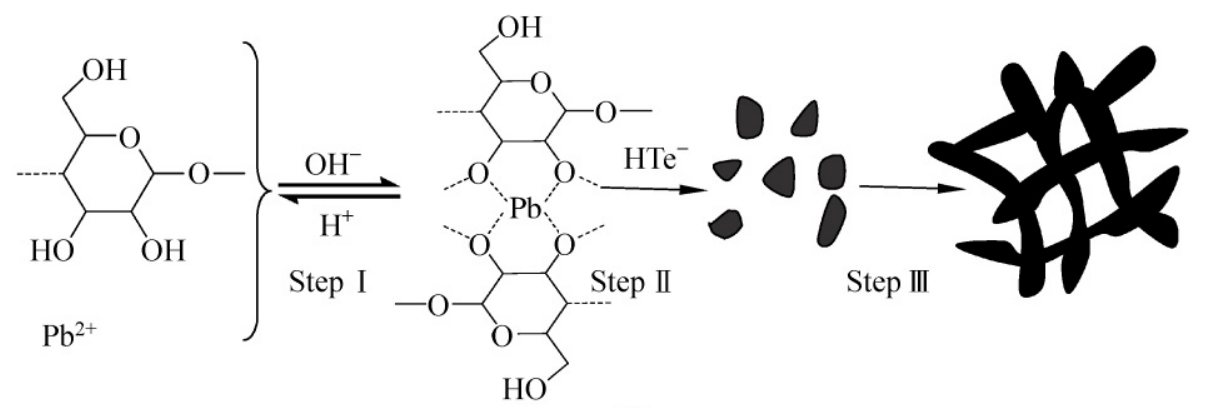

(a)

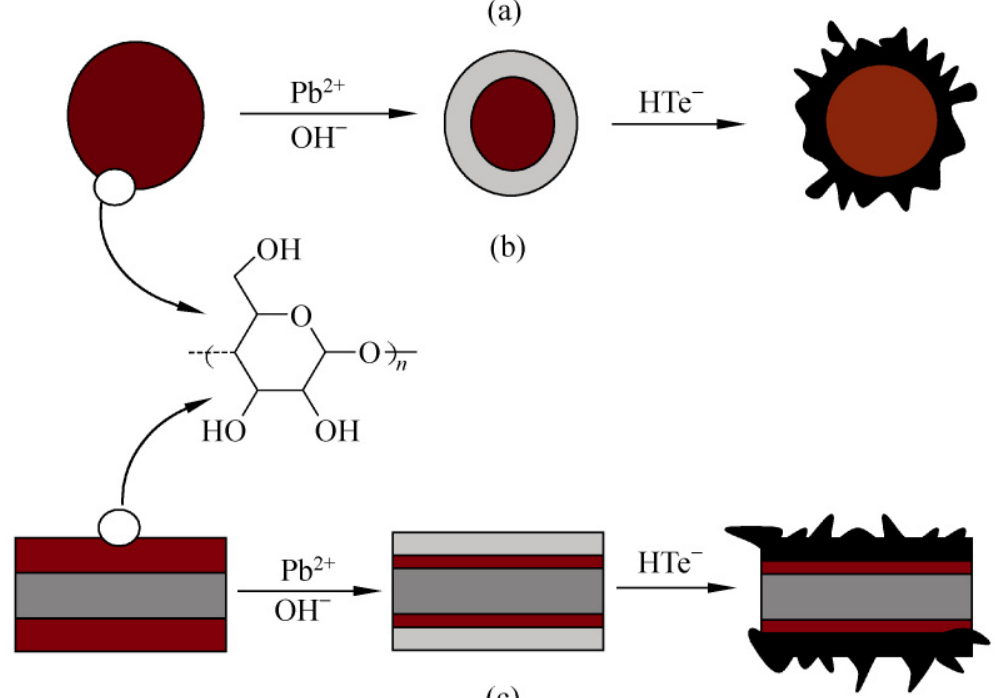

(c)

Figure 4 Schematic illustration of the formation of PbTe nanoparticle networks in aqueous media containing carbohydrates (a), carbohydrate spheres@PbTe core shell structures (b), and Te@carbohydrate tubes@PbTe multilayer cable structures (c) 
(Step II) of the lead polyolato complexes with $\mathrm{NaHTe}$ produces $\mathrm{PbTe}$ clusters or nanoparticles stabilized by the deprotonated carbohydrates. This is consistent with the TEM image (Fig. S-15 in the ESM) of the sample produced in an extremely dilute solution. However, unlike trioctylphosphine oxide (TOPO), oleic acid, and other commonly used organic capping agents, carbohydrates have a much weaker stabilization capability, which prevents the formation of highly dispersed nanoparticles. This allows the clusters or nanoparticles to form more stable continuous nanoparticle networks (Step III), leading to a reduction in the surface energy. Since PbTe has the highly symmetric cubic rocksalt crystal structure, the absence of dipoledipole interactions leads to a random orientation of nanoparticles in the networks, as shown by the TEM image in Fig. 1(e).

In addition to $\mathrm{PbTe}$, other semiconductor networks including $\mathrm{PbSe}$ and $\mathrm{Bi}_{2} \mathrm{Te}_{3}$ can be produced using a similar strategy. Representative TEM images and XRD patterns shown in Fig. 5 confirm the successful synthesis of $\mathrm{PbSe}$ and $\mathrm{B}_{2} \mathrm{Te}_{3}$ networks when $\beta$-cyclodextrin and EDTA, respectively, were employed as ligands.

\section{Conclusions}

We have developed a novel one-step approach for the synthesis of nanocrystalline semiconductor networks using weak ligands. As illustrated for PbTe networks, a variety of carbohydrates can act as weak ligands which facilitate the formation of continuous nanoparticle networks. The nanoparticle size can be tuned by changing the temperature and concentration. Through a similar strategy, more complicated structures including spherical core-shell structures and multilayered submicron cables have been fabricated. This approach is general and can be readily extended to the

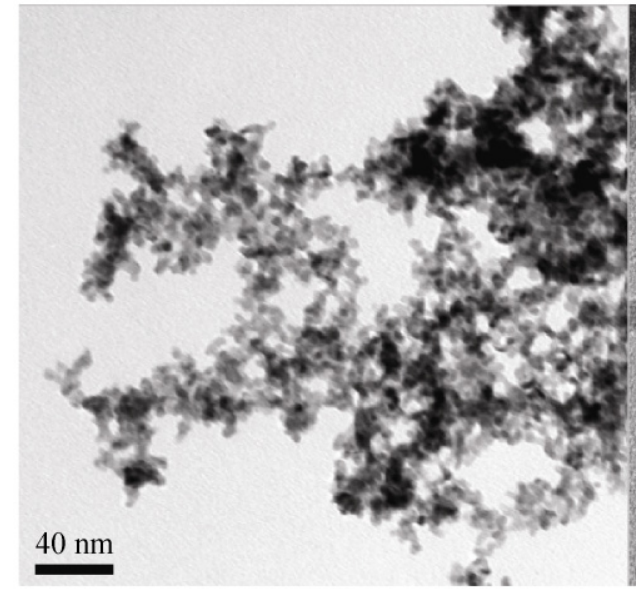

(a)

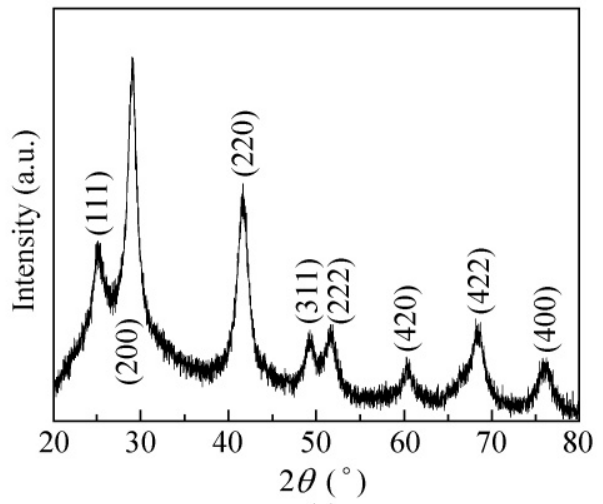

(c)

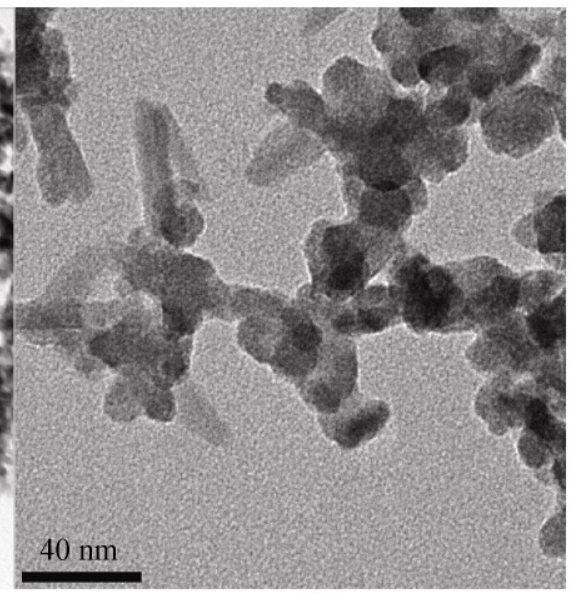

(b)

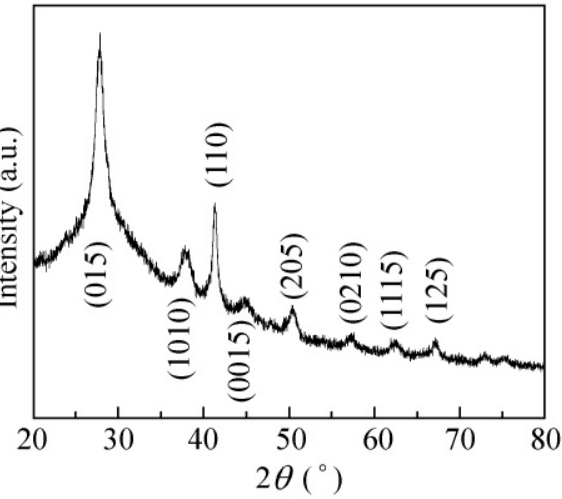

(d)

Figure 5 (a) TEM image and (c) XRD pattern of a nanocrystalline PbSe network prepared using $\beta$-cyclodextrin as a ligand. (b) TEM image and (d) XRD pattern of a nanocrystalline $\mathrm{Bi}_{2} \mathrm{Te}_{3}$ network prepared using EDTA as a ligand 
fabrication of other semiconductor networks, including $\mathrm{PbSe}$ and $\mathrm{Bi}_{2} \mathrm{Te}_{3}$.

\section{Acknowledgements}

This work was supported by Toyota Motor Engineering and Manufacturing North America.

Electronic Supplementary Material: EDS spectra, FTIR spectra, XPS spectra, and TEM images of PbTe nanoparticle networks using a variety of ligands under different conditions and EDS spectra and XRD patterns of carbohydrate spheres@PbTe core-shell composites and Te@carbohydrate tubes@PbTe multilayer submicrocables are available in the online version of this article at http://dx.doi.org/10.1007/s12274-010-0030-8 and accessible free of charge.

Open Access: This article is distributed under the terms of the Creative Commons Attribution Noncommercial License which permits any noncommercial use, distribution, and reproduction in any medium, provided the original author(s) and source are credited.

\section{References}

[1] Alivisatos, A. P. Semiconductor clusters, nanocrystals, and quantum dots. Science 1996, 271, 933-937.

[2] Peng, X. G. An essay on synthetic chemistry of colloidal nanocrystals. Nano Res. 2009, 2, 425-447.

[3] Jun, Y. W.; Choi, J. S.; Cheon, J. Shape control of semiconductor and metal oxide nanocrystals through nonhydrolytic colloidal routes. Angew. Chem. Int. Ed. 2006, 45, 3414-3439.

[4] Par, J.; Joo, J.; Kwon, S. G.; Jang, Y.; Hyeon, T. Synthesis of monodisperse spherical nanocrystals. Angew. Chem. Int. Ed. 2007, 46, 4630- 4660.

[5] Burda, C.; Chen, X. B.; Naryanna, R.; El-Sayed, M. A. Chemistry and properties of nanocrystals of different shapes. Chem. Rev. 2005, 105, 1025-1102.

[6] Korgel, B. A.; Fitzmaurice, D. Self-assembly of silver nanocrystals into two-dimensional nanowire arrays. Adv. Mater. 1998, 10, 661-665.

[7] Pacholski, C.; Kornowski, A.; Weller, H. Self-assembly of $\mathrm{ZnO}$ : From nanodots to nanorods. Angew. Chem. Int. Ed. 2002, 41, 1188-1191.

[8] Yu, J. H.; Joo, J.; Park, H. M.; Baik, S.; Kim, Y. W.; Kim, S. C.; Hyeon, T. Synthesis of quantum-sized cubic $\mathrm{ZnS}$ nanorods by the oriented attachment mechanism. J. Am. Chem. Soc. 2005, 127, 5662-5670.

[9] Cho, K. S.; Talapin, D. V.; Gaschler, W.; Murray, C. B. Designing PbSe nanowires and nanorings through oriented attachment of nanoparticles. J. Am. Chem. Soc. 2005, 127, 7140-7147.

[10] Tai, G. A.; Guo, W. L.; Zhang, Z. H. Hydrothermal synthesis and thermoelectric transport properties of uniform singlecrystalline pearl-necklace-shaped $\mathrm{PbTe}$ nanowires. Cryst. Growth Des. 2008, 8, 2906-2911.

[11] Tang, Z. Y.; Kotov, N. A.; Giersig, M. Spontaneous organization of single CdTe nanoparticles into luminescent nanowires. Science 2002, 297, 237-240.

[12] Kim, F.; Kwan, S.; Akana, J.; Yang, P. Langmuir-Blodgett nanorod assembly. J. Am. Chem. Soc. 2001, 123, 4360-4361.

[13] Xu, X. X.; Wang, X.; Nisar, A.; Liang, X.; Zhuang, J.; Hu, S.; Zhuang, Y. Combinatorial hierarchically ordered 2D architectures self-assembled from nanocrystal building blocks. Adv. Mater. 2008, 20, 3702-3708.

[14] Murray, C. B.; Kagan, C. R.; Bawendi, M. G. Self-organization of CdSe nanocrystallites into 3-dimensional quantum-dot superlattices. Science 1995, 270, 1335-1338.

[15] Nykypanchuk, D.; Maye, M. M.; van der Lelie, D.; Gang, O. DNA-guided crystallization of colloidal nanoparticles. Nature 2008, 451, 549-552.

[16] Park, S. Y.; Lytton-Jean, A. K. R.; Lee, B.; Weigand, S.; Schatz, G. C.; Mirkin, C. A. DNA-programmable nanoparticle crystallization. Nature 2008, 451, 553-556.

[17] Ahniyaz, A.; Sakamoto, Y.; Bergstro, L. Magnetic fieldinduced assembly of oriented superlattice from maghemite nanocubes. Proc. Nat. Acad. Sci. USA 2007, 104, 1757017574.

[18] Redl, F. X.; Cho, K. S.; Murray, C. B.; O’Brien, S. Threedimensional binary superlattices of magnetic nanocrystals and semiconductor quantum dots. Nature 2003, 423, 968-971.

[19] Shevchenko, E. V.; Talapin, D. V.; Murray, C. B.; O’Brien, S. Structural characterization of self-assembled multifunctional binary nanoparticle superlattices. J. Am. Chem. Soc. 2006, 128, 3620-3637.

[20] Zhang, Y. X.; Zeng, H. C. Gold sponges prepared via hydrothermally activated self-assembly of Au nanoparticles. J. Phys. Chem. C 2007, 111, 6970-6975.

[21] Mohanan, J. L.; Arachchige, U. I.; Brock, S. L. Porous semiconductor chalcogenide aerogels. Science 2005, 307, 397-400.

[22] Arachchige, U. I.; Brock, S. L. Sol-gel assembly of CdSe nanoparticles to form porous aerogel networks. J. Am. Chem. Soc. 2006, 128, 7964-7971. 
[23] Hanrath, T.; Veldman, D.; Choi, J. J.; Christova, C. G.; Wienk, M. M.; Janssen, R. A. J. PbSe nanocrystal network formation during pyridine ligand displacement. ACS Appl. Mater. Interfaces 2009, 1, 244-250.

[24] Urban, J. J.; Talapin, D. V.; Shevchenko, E. V.; Murray, C. B. Self-assembly of PbTe quantum dots into nanocrystal superlattices and glassy films. J. Am. Chem. Soc. 2006, 128, 3248-3255.

[25] Murphy, J. E.; Beard, M. C.; Norman, A. G.; Ahrenkiel, S. P.; Johnson, J. C.; Yu, P.; Micic, O. I.; Ellingson, R. J.; Nozik, A. J. PbTe colloidal nanocrystals: Synthesis, characterization, and multiple exciton generation. J. Am. Chem. Soc. 2006, 128, 3241-3247.

[26] Dughaish, Z. H. Lead telluride as a thermoelectric material for thermoelectric power generation. Physica B 2002, 322, 205-223.

[27] Sootsman, J. R.; Kong, H.; Uher, C.; D'Angelo, J. J.; Wu, C. I.; Hogan T. P.; Caillat, T.; Kanatzidis, M. G. Large enhancements in the thermoelectric power factor of bulk $\mathrm{PbTe}$ at high temperature by synergistic nanostructuring. Angew. Chem. Int. Ed. 2008, 45, 8618-8622.

[28] Zhang, H.; Zhou, Z.; Yang, B. The influence of carboxyl groups on the photoluminescence of mercaptocarboxylic acid-stabilized CdTe nanoparticles. J. Phys. Chem. B 2003, $107,8-13$.

[29] Wang, Q.; Li, H.; Chen, L. Q.; Huang, X. J. Monodispersed hard carbon spherules with uniform nanopores. Carbon 2001, 39, 2211-2214.

[30] Yang, R. Z.; Qiu, X. P.; Zhang, H. R.; Li, J. Q.; Zhu, W. T.; Wang, Z. X.; Huang, X. J.; Chen, L. Q. Monodispersed hard carbon spherules as a catalyst support for the electrooxidation of methanol. Carbon 2005, 43, 11-16.

[31] Sun, X. M.; Li, Y. D. $\mathrm{Ga}_{2} \mathrm{O}_{3}$ and $\mathrm{GaN}$ semiconductor hollow spheres. Angew. Chem. Int. Ed. 2004, 43, 3827-3831.

[32] Sun, X. M.; Li, Y. D. Colloidal carbon spheres and their core/shell structures with noble-metal nanoparticles. Angew. Chem. Int. Ed. 2004, 43, 597-601.

[33] Qian, H. S.; Yu, S. H.; Luo, L. B.; Gong, J. Y.; Fei, L. F.; Liu, X. M. Synthesis of uniform Te@carbon-rich composite nanocables with photoluminescence properties and carbonaceous nanofibers by the hydrothermal carbonization of glucose. Chem. Mater. 2006, 18, 2102-2108.

[34] Mays, C. W.; Vermaak, J. S.; Kuhlmann-Wilsdorf, D. On surface stress and surface tension. II. Determination of the surface stress of gold. Surf. Sci. 1968, 12, 134-140.

[35] Tong, H.; Zhu, Y. J.; Yang, L. X.; Li, L.; Zhang, L. Lead chalcogenide nanotubes synthesized by biomolecule-assisted self-assembly of nanocrystals at room temperature. Angew. Chem. Int. Ed. 2006, 45, 7739-7742.

[36] Klufers, P.; Schulhmacher, J. Sixteenfold deprotonated $\gamma$-cyclodextrin tori as anions in a hexadecanuclear lead(II) alkoxide. Angew. Chem. Int. Ed. 1994, 33, 1863-1865. 